\title{
How can healthcare organisations enhance their capacity to learn?
}

\author{
Compiled by Tom Smith
}

- What is the influence of culture on the ability of health services to learn?

- Why do hospitals fail to learn from errors?

- How will advances in technology such as telemedicine help to spread learning?

These and other questions are raised in the papers outlined below.

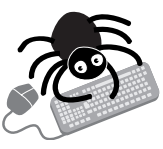

Please visit the QSHC website

(http://www.qshc.com) for links to these articles - many to full text.

Learning about culture Organizational culture is often used to explain extraordinary organizational performance. In fact, the term "safety culture" has recently emerged in the healthcare literature to describe the set of assumptions and practices necessary for healthcare organizations to provide optimal care. More and more, the benefits of shared culture are talked about in health care. But, as noted in the first paper below, shared "norms and assumptions can also be a source of danger if they blind the collective to vital issues or factors important to performance that lie outside the bounds of organizational perception. Cultural blind spots can lead an organization down the wrong path, sometimes with dire performance consequences. This was the case at the Bristol Royal Infirmary (BRI).

What can we learn about the role of culture in health care from Bristol? - Karl Weick is one of the most respected academic figures in organisational behaviour. He is the author of Sensemaking in Organizations (1995) and a former editor of Administrative Science Quarterly. His paper is focused on the question: "Why did Bristol Royal Infirmary continue to perform pediatric cardiac surgeries for almost 14 years (1981-95) in the face of poor performance?".

"The working definition of culture used in the BRI inquiry was 'those attitudes, assumptions, and values which condition the way in which individuals and the organization work'. The BRI board of inquiry variously referred to the BRI as a provider-oriented culture, a culture of blame, a club culture, a culture of fear, an oral culture, a culture of justification, a culture of paternalism, and a culture of uncertainty. Bristol is described as a collection of fragmented, loosely coupled, self-contained subcultures (the inquiry board calls them 'tribes'), managed by a CEO whose idea of leadership and oversight was to say, 'You fix it'. The BRI culture is one in which people share the practice of explaining or justifying . . . mediocre or poor results on the basis of case severity rather than directing attention to producing better results." The paper argues that the problem at BRI was "'cultural entrapment' - the process by which people get locked into lines of action, subsequently justify those lines of action, and search for confirmation that they are doing what they should be doing".

"When people take important actions that are visible and hard to undo, it is hard for them to deny that the actions actually occurred. Public, irrevocable, chosen actions put reputations on the line and compel some kind of explanation and justification. It is important to understand that the idea of justification as used here is not synonymous with mere individual self-justification or defensiveness. Justification is rationalizing done within socially acceptable bounds. Rationalization will not work unless it is culturally appropriate. The content of those justifications is not chosen casually because so much is at stake. Only a limited number of justifications are socially acceptable, and people have to live with the justifications they adopt. Thus, whatever justifications people voice tend to have considerable tenacity, they tend to influence subsequent perceptions and action, and they focus disproportionately on information that confirms their validity rather than disconfirms it. These ideas help us make sense of what happened at BRI."

"Tenacious justifications make it harder to learn, harder to discontinue the justified action, and easier to spot information that confirms their validity. This basic social process for constructing reality is common to organizations of all kinds, both those experiencing adversity and those experiencing success. BRI reconstructed a history of excess deaths and transformed it into a history of excess complexity. The reconstructed rationale persists because layers of bureaucrats above the surgical unit, people who had some say in the original choice to designate BRI as a center of excellence, find their own judgments in jeopardy. The unintended consequence is that the whole chain of decision makers comes to support an explanation that makes it difficult for an underperforming unit to improve or to stop altogether."

What is Weick saying should change? "If there is a maxim implied in this analysis, it reads 'challenge easy explanations'. An 'easy' explanation is one that that has shallow plausibility, meaning that it can explain away any outcome, is not readily refuted, and the best that can be done to disarm it is to doubt it. Easy explanations for the poor outcomes at BRI included: 'our poor outcomes will improve over time with experience', 'outcomes will improve once we get a hoped-for new surgeon', and 'our poor outcomes are an artifact of small numbers that look worse when converted into percentages, and they are inevitable because we are treating sicker children'. As the board of inquiry said: 'All of these arguments had sufficient plausibility at the time that they could be believed, and they could not be readily refuted, though they might be doubted'. Justification turns a conspicuous action into a meaningful action. The resulting meaning can promote or impede improvement." The conclusion is that socially acceptable reasons that are available for justification must be placed at the centre of a learning orientated analysis "that values communication, openness, mutual aid, and mindful attention to patient care".

People in the sharp end of practice "are torn between justification and candor. Their public irrevocable acts tempt them to justify, but their forced compliance with directives from above tempts them toward candor. The tensions created by these opposing temptations may mean that frontline medical workers are people at a tipping point. That possibility is important because it means that they may welcome surprisingly small interventions of support, security, and psychological safety that could tip the balance toward candor and learning and away from concealment and justification." Without these, "hospitals are contexts in which autonomy works against learning. The net result is that change is next to impossible."

Individual interpretations must be open to collective probing. "The unwillingness and inability to see and improve interdependence at BRI was the feature most often criticized. When people understand interdependence, behavioral commitment can be moderated. What began as merely a plausible justification is likely to harden into dogma because it performs such an important function. Dogma precludes learning, and it precludes improvement. This is what happened at BRI and it need not happen again."

$\Delta$ Weick K, Sutcliffe K. Hospitals as cultures of entrapment: a re-analysis of the Bristol Royal Infirmary. Calif Manage Rev 2003;45:73-84. 
Why do hospitals fail to learn? The authors begin by stating: "the importance of hospitals learning from their failures hardly needs to be stated. Not only are matters of life and death at stake on a daily basis, but also an increasing number of US hospitals are operating in the red." Learning is "imperative" to address both clinical and organizational problems. The authors conducted "a detailed study of hospital nursing care processes [they observed 26 nurses for 239 hours in nine hospitals] to investigate conditions under which nurses might respond to failures they encounter in their hospital's operational processes by actively seeking to prevent future occurrences of similar failures". They found that "process failures are not rare but rather are an integral part of working on the front lines of health care delivery".

The authors focus on "problems" rather than "error" for two reasons. First, "the relative visibility and frequency of problems, compared to errors, makes them accessible to front-line workers who are well positioned to suggest important changes that managers would not be able to identify. Second, problems carry less stigma than errors, making discussion of them less interpersonally threatening." They distinguish between two learning approaches to problem solving: (1) short term fixes and (2) changing underlying routines to prevent problems occurring. The latter is held to be more effective for long term learning.

Nurses experienced five broad types of problems: (1) missing or incorrect information; (2) missing or broken equipment; (3) waiting for a (human or equipment) resource; (4) missing or incorrect supplies; and (5) simultaneous demands on their time. They found that, in spite of increased emphasis on resolving underlying causes, "hospitals are not learning from the daily problems and errors encountered by their workers". So "why aren't hospitalsand [the authors suspect] many other service organizations as well-learning all they can from daily problems encountered by their workers?" The authors dismiss two explanations, that it might be because "problems are highly complex or difficult to solve" or "nurses are unmotivated".

"The lack of organizational learning from failures can be explained instead by three less obvious, even counterintuitive, reasons:

(1) An emphasis on individual vigilance in health care: each caregiver thus tends to work on completing her or his own tasks without altering common underlying processes.

(2) Unit efficiency concerns: nursing labor is expensive and in short supply. Understandably, hospitals can ill afford to have nurses routinely working with slack resources. This staffing model leads to an organizational design where workers do not have time to resolve underlying causes of problems that arise in daily activities. Instead, nurses are barely able to keep up with the required responsibilities and are in essence forced to quickly patch problems.

(3) A widely shared goal of developing units that can function without direct managerial assistance: reducing the degree to which managers are available to front-line staff can be a loss for improvement efforts, especially when workers are already overburdened by existing duties."

"These three factors, while seemingly beneficial for nurses and patients alike, can ironically leave nurses under-supported and overwhelmed in a system bound to have breakdowns. In most hospitals, organizational culture and management behaviors tend to reinforce this already-robust system of individual vigilance. Seventy percent of the nurses we interviewed commented that they believed their manager expected them to work through the daily disruptions on their own. Speaking up about a problem or asking for help was likely to be seen as a sign of incompetence. Workers experience an increasing sense of frustration, exhaustion and, in some cases, leave the organization-worn out by the task of swimming upstream against an incessant tide of small, annoying problems. Across the healthcare delivery industry, this phenomenon is contributing to unacceptably high levels of turnover in many organizations and to widespread nursing shortages tide of small, annoying problems."

There are two things managers can do to "lever learning":

- provide more supportive advice, assistance and focus on the management of patient care;

- create an environment in which people can speak openly about problems.
"Are these solutions feasible in the budget conscious world of health care? After all, most involve additional expenses, whether freeing up a manager to assist front-line workers with resolving failures, promoting more discussion of (and time devoted to) tracking down causes of problems, or implementing counter measures. Further analysis suggests that the extra expense would pay off. Although second-order problem solving requires an investment in developing both human resources and organizational routines, over time the reduction in failures could pay for themselves."

A Tucker A, Edmondson A. Why hospitals don't learn from failures: organizational and psychological dynamics that inhibit system change. Calif Manage Rev 2003;45:55-72

\section{Learning across managerial and} professional domains One of the key relationships in organisational learning is trust between management and professionals. This year a number of articles have emerged on relationships between management and professional perspectives and the tensions and opportunities these provide for learning across domains. The kind of frustrations expressed above-that management is not always sensitive to nursing views-led to a strike in Minnesota which forms part of the discussion in an article by Preuss and Frost in California Management Review.

The waxing and waning of cooperation - The paper examines the "waxing and waning" of "labor-management cooperation" with a case study of health care, exploring relationships between management and a nursing union from the mid 1980s until the late 1990s in the US "twin cities" of Minneapolis and St Paul, Minnesota.

The key impact on traditional forms of cooperation came from managed care of which the twin cities were an early adopter; "by $199244 \%$ were enrolled in an $\mathrm{HMO}$, one of the highest levels of ... penetration in the country". "As hospitals are high-cost sites of health care delivery, HMOs seek to limit physicians' use of hospital services and the average length of stay. The result was growing pressure on hospitals to alter patient care delivery and overall cost structures. In contrast to other regions in the country, union density in the health sector has historically been very high in the twin cities. In 1989, the Minnesota Nurses Association (MNA) represented $91 \%$ of all registered nurses." This unusually high rate means that the character of labour-management relations had been closer to the UK model with "the hospitals and unions negotiating area-wide contracts to standardize employment costs". In 1984 the hospitals and the MNA sat down to negotiate a new contract "in the context of the dramatic external changes then taking place". Nurses wished to exercise some control over the basis on which staff were being made redundant. Failure to agree saw "the largest nursing strike in US history" with 6300 nurses "striking 16 hospitals for 39 days".

Over the next 5 years hospital systems merged and "in 1989, corporate human resource managers from several hospitals and union leaders that were jointly engaged in labor-management cooperation came together to form the Metropolitan Hospitals' Labor-Management Council (MHLMC) to promote the expansion of" relations. The strength of this arrangement "was highlighted soon after" in 1991 when a large hospital closed and "with close collaboration, nearly $90 \%$ of the 1475 employees were placed in new jobs". But greater openness never fully replaced traditional relations and would diminish in the months leading to negotiations with each side giving out "less information". "Moreover, the trust broken during adversarial negotiations would always need repair." Both sides wanted openness to win out and in 1995 it was agreed each MHLMC hospital would have a local labour-management committee. The variation adopted locally "would later create tensions" among hospitals and the union.

In the face of increasing competition, hospitals found it difficult to balance cooperation between each other on the negotiating committee with competition with each as alternative providers and employers. "Hospital administrators wanted the flexibility to negotiate contracts that reflected their competitive needs. As a result, many ... felt they spent more time negotiating with each other than with the MNA." Some wanted to raise wages to secure staff, others worried about the knock-on effect. "As hospitals diverged in 
their strategic approaches, hospitals that preferred arms' length relations did not want others with more extensive processes limiting their actions".

"The final straw came with the privatisation of the University of Minnesota hospital system and its sale to another system in 1997". The new owners forced an election process to regain union representation rights. The union asked other employers in the MHLMC to put pressure on the new employer. When they failed to do so the union saw it as a "clear test of the value employers placed on citywide cooperation". After several months of stalemate, the MNA withdrew, leading to the break up of the committee.

Contracts had diverged to the point of varying wage levels and members of the same union having different powers to set staffing levels and restrict patients admitted to units. Over 13 years, the relatively unified bargaining systems had broken up leaving neither side with the view that collective bargaining could be beneficial. The authors suggest that, increasingly, "cooperation may be but a short-lived phenomenon. Often after a period of initial success, cooperative efforts find themselves bogged down, no longer meeting one or more of the parties' needs, or held hostage to events taking place elsewhere in the parties' relationship."

"Realizing that conflict does not go away when parties enter into cooperative undertakings is a large step towards managing the tensions that inevitably arise. Union representatives and managers must realize that the existence of cooperation does not eliminate conflict. Further, they need to know that conflict is not pathological, but rather is a natural outcome of such a relationship." The authors say that, despite the demise of cooperation, the benefits accrued through cooperation are clear. The suggestion is that cooperation is cyclical and will re-emerge at the point when inherent conflict cannot be resolved locally.

$\Delta$ Preuss G, Frost A. The rise and decline of labor-management cooperation: lessons from health care in the twin cities. Calif Manage Rev

2003:45:85-106

International tensions in manager-professional relations - It is not only tensions with nurses that have been addressed. The $B M$, for example, had a special issue on management-doctor relationships in March, with one article suggesting that heightened tensions in the UK can be located in international trends.

Doctor-management relations are largely shaped "by the changing structural arrangements from within which health care is delivered". Changes introduced in Britain match trends in other countries - the strengthening of management, introduction of (quasi) market mechanisms, and more focused performance and accountability initiatives.

These have inevitably led to changes in doctor manager relations. "Managers increasingly had a corporate outlook, seeking to maintain and develop their own institutions' facilities." "There has been an increasing 'systematisation' of medical knowledge." This has occurred "partly through methods that allow the measurement of medical work for managerial purposes-for example, case-mix measures such as diagnostic related groups" and partly through "strategies that determine how doctors should deliver care, both at the level of the individual patient encounter (for example, clinical protocols and guidelines) and in terms of the processing of patients through the system". Increased attention has been paid to financial incentives and state regulation has been strengthened. "An overriding theme of the diverse changes over 30 years has been a substantial erosion of the professional medical dominance and autonomy."

"If, as the Government hopes, regulated markets and consumer choice are to be effective forces for improved quality and performance, then this cannot happen without better alignment between doctors and the organizations in which they provide services. The extent of cultural divergence between managers, doctors and other professional groups suggests that such realignment will be far from easy."

$\Delta$ Davies $\mathbf{H}$, Harrison S. Trends in doctor manager relationships. BM 2003;326:646-9

-An editorial co-written by doctors and managers points to the inherent differences in perspectives between the two. For example, "doctors worry about patient outcomes managers . . . about patient experience". Patients are "best served by a tension between the two". Simplistic notions are put forward to improve relations - for example, that management should not be involved in clinical issues. But "this argument ignores the mounting body of evidence that badly managed organizations fail patients, frustrate staff [and] deliver poor quality care". Another argument is to "make managers think and behave like doctors or vice versa". But, while closer relations between thinking would help, difference is desirable: "each group has a unique contribution which needs to be valued and respected". In developing better understanding between the groups and engaging with difficult tensions between them, policy and other agencies should ensure "their approach to planning and performance management does not add tensions but allows space for doctors and managers to agree shared objectives".

\ Edwards N, Marshall M, McLellan A, Abbasi K. Doctors and managers: a problem without a solution? BMV 2003;326:609-10

The need for management to institutionalise a quality culture - Increased talk of quality has not caused mainstream organisational activity to be related to this aim. If staff are to be concerned with quality improvement, "these roles must be embedded in structures that support the requisite information flows and specify the appropriate scope of responsibility and authority for interdependent positions".

In comparison with other writers, Adler et al are "less focused on 'change agents' and 'champions' because we believe their effectiveness depends critically on the broader organizational context within which they function. The attention devoted to these roles reflects, we believe, excessive cynicism about the organizational context and human nature. Change agents and champions are pushed to center stage in many accounts because they are seen as heroes in a constant struggle for innovation against the stifling effects of bureaucracy and people's tendency to resist change."

They want to see an explicit organisational focus on clinical care improvement. Decentralised department based organisation does not support quality improvement aims. "In one instance we studied, an asthma pathway development project was in limbo for a full year because three physicians could not come to agreement on it. Disagreement is healthy, but the structure within which these physicians functioned allowed them to stall a major project. They could have been challenged to work on the problem continuously until they reached a workable compromise."

A study of seven hospitals found those that "strengthened the structural components of their improvement capability had more rigorously standardized processes, and more oversight and accountability mechanisms that were developed through-and supported-a process of extensive dialogue between hospital and medical staff and across the layers of authority and status".

$\Delta$ Adler P, Riley P, et al. Performance improvement capability: keys to accelerating performance improvement in hospitals. Calif Manage Rev 2003;45: 12-33

\section{Learning through information Techno-} logical advances in health care could drive learning that transcends organisational boundaries but, as the following article by Amy Edmondson stresses, cannot be considered in isolation from organisation or cultural issues

Framing learning in organisations - Amy Edmondson, who co-wrote the paper with Tucker on why hospitals fail to learn, examines technology transfer in this second paper and argues that developing an understanding of the social dimension of learning is critical to successful implementation. What is often not appreciated is that "many new technologies disrupt existing organizational routines and relationships, requiring potential users to re-learn how to work together-a challenge that usually proves more difficult than anticipated".

"What is clear from prior research is that technology implementation is difficult-and especially difficult when an innovation challenges existing patterns of interdependence among individuals or groups. In these cases, implementation becomes an organizational learning challenge in which shared perceptions about organizational risks and benefits may be as important as technical advantages to implementation success."

"Simply by working closely together, people tend to develop shared assumptions and beliefs - a process sometimes referred to 
as 'sensemaking' or 'social construction of reality'" (she prefers the phrase "framing" reality). A frame is a set of assumptions and beliefs about a particular object or situation and the process of framing is a process of creating meaning-either passively and unconsciously or actively and consciously. Framing is neither bad nor good; it is simply inevitable. We interpret what is going on around us through a lens shaped by our personal history and our current social context. The catch is, we tend to assume that our framing captures the truth, rather than presenting a subjective "map" of territory that could instead be mapped differently. "By becoming aware of and altering these skewed frames, organizational participants can learn more and achieve better results."

Edmondson is of the view that leadership is critical to reframing. "First, a project leader is the organization's lead user-the most visible spokesperson and translator of the potential implications of a new technology for the organization. Second, research has shown that people pay particular attention to what leaders say and do, compared to what peers and others say and do."

In exploring the impact of leaders, she discusses the experience of four teams adopting a new less invasive surgical procedure. Successful adoption requires the team to work in a new way. "Two of the four teams ... were successful implementers; two were not. Two had senior surgeons in charge, and two had newer, junior surgeons. Two were academic medical centers; two were community hospitals. Two had more management support; two had less. None of these factors explained the differences in implementation success that emerged."

"Three dimensions or themes emerged as characterizing differences in how implementation was framed: project purpose, the leaders' role, and the team's role. The successful leaders saw the innovation as a chance for the team to learn together and were involved, the two places that dropped the new technique had leaders who remained separate from the team. The two successful team leaders 'framed their role in the project differently than [the less successful]. Specifically, they explicitly communicated their inter-dependence with others, emphasizing their own fallibility and need for others' input. Without conveying any loss of expertise or status, these leaders simply recognized (and communicated) that they were dependent on others. At the unsuccessful sites, team members 'struggled with the changes that the new technology required of them, particularly in the face of the surgeons' lack of acknowledgement of significant and profound change'. In contrast, successful 'teams had a palpable sense of teamwork and collegiality . . . aided by early practice sessions in which each team conducted a dry run using the new technology."

"Taken together, the sites that had an aspirational purpose, interdependent team leaders, and empowered teams represent a 'learning frame'. Those projects in which the goals were defensive, and in which leaders were seen as technical experts and the rest of the teams as supporting doers, can be characterized instead as having a 'performance frame'."

"New technologies often change work processes in organizations and correspondingly, require new roles to enact them. Yet, to realize creative new possibilities, group roles must be reframed. Promised benefits in quality or efficiency may be difficult to realize unless users learn to how to work differently as interdependent members of a complex system."

$\Delta$ Edmondson A. Framing for learning: lessons in successful technology implementation. Calif Manage Rev 2003;45:35-54.

Conclusions the papers reviewed here are concerned with ways to improve learning in organisations and are written in relation to health care. Some are concerned with culture and the sharing of meaning in groups, others with ways in which reflection can be better structured within organisations, and a third group with information and its capacity to inform better practice. In each approach learning is shown to be a critical function of group dynamics and communication between people.

T Smith

Research Associate, Judge Institute of Management, Cambridge University, Cambridge CB2 1AG, UK ts271@cam.ac.uk 